\title{
Breaking Barriers on Reuse of Digital Devices Ensuring Final Recycling
}

\author{
David Franquesa, Leandro Navarro, David López, Xavier Bustamante, Santiago Lamora \\ Department of Computer Architecture \\ Universitat Politècnica de Catalunya \\ Barcelona, Spain \\ Email: \{david.franquesa, leandro, david, xavier.bustamante, slamora\}@ac.upc.edu
}

\begin{abstract}
Digital Devices or Electric and Electronic Equipment (EEE) are scrapped at an alarming rate instead of being salvaged, fixed, and reused. For the reuse sector to flourish, donors, receivers, and reuse centers need services and technologies to gain effectiveness, efficiency, and traceability to reach the goal of greatly extending the lifetime of devices and still ensuring their final recycling. The main challenges to overcome are access to sufficient good-quality used devices, quick preparation of those with greatest potential for reuse, ability to make direct donations, guarantee that reused devices are eventually recycled, communication of the social and environmental value of reuse, establishing a system to reward donors, and ensuring commitment of receivers of reused devices to recycling. We present a set of open-source tools based on a distributed platform ecosystem that supports direct donation of devices. Devices are prepared for reuse in the donor's location, and receivers collect them. Malicious users are discouraged by a reputation scheme to reward cooperative receivers that reuse devices and track them until disposed to recycling agents. This reduces costs, and minimizes EEE losses as there is no need for a central logistic system or centralizing the engagement of donors on charity projects. The background and foreground Intellectual Property follows an open model (unrestricted), as the goal is to bootstrap the reuse process, generate local efficiencies, guarantee final recycling, and ensure traceability. Pilots already performed for more than four years and two thousand devices validate the model with $80 \%$ traceability of device components.
\end{abstract}

\section{INTRODUCTION}

Many digital devices — such as desktop, laptop, tablet, or mobile computers - from businesses and public organisations are dismantled and recycled when being amortized or out of guarantee, despite being nearly up-to-date and in perfect condition.

The latest research on e-waste estimates that about 41.8 million metric tonnes (Mt) of e-waste was generated in 2014 and that this number will increase to $50 \mathrm{Mt}$ as early as 2018. In Europe, the total e-waste generation was $11.6 \mathrm{Mt}$ in 2014. The European countries with the highest e-waste generation in absolute quantities are Germany $(1.8 \mathrm{Mt})$, the United Kingdom (1.5 Mt), and France (1.4 Mt). In 2012, only 3.2 Mt of e-waste was officially collected in the 28 member states of the European Union, whereas 9 Mt of e-waste was generated in 2012 in this region. The current practices for dealing with locally generated WEEE (Waste Electric and Electronic Equipment) seem unsatisfactory, because they lead to a loss of secondary resources and damage the environment
[1]. Alternatives to mitigate the production of e-waste come from reduction and reuse.

Reuse of digital devices ensures recycling, effectively contributes to generating a circular economy, prevents waste generation, reduces the risk of WEEE issues such as leakage to landfills or illegal exports, creates jobs, and strengthens the digital skills [2]. Reuse can also help to reduce the digital divide and strengthen institutions and projects for social change. However, why is it such a minority practice in Europe? When companies, governments, or individuals need to get rid of their digital devices to reuse, they don't know where to turn. This results in most EEE being recycled too early, despite the explicit demand for reuse coming from social and charity organisations.

Even though it is a paradox that the volume of e-waste is growing three times faster than other types, recycling plants collect less and less e-waste. This is mainly attributed to pillaging on collection points or by home collection done by unauthorized operators [3]. Pilots performed and presented on this paper confirm that in public institutions more than $80 \%$ of the digital devices to be recycled are operational, and there is a demand for these devices without any upgrade or repair needed. So in countries with a crisis situation, such as Spain, recycling waste (products) with potential to be reused is not effective. In Europe, only 33\% of EEE Waste is reported as collected and treated. The remaining is a leakage: 13\% goes to EU landfill and 54\% to substandard treatment inside and outside the EU (illegal trade to Third World countries) [5]. According to the International Environmental Technology Center of the United Nations Environment Program (UNEP) [7], e-waste is the fastest-growing type of waste, particularly in some developing countries where the volume is expected to grow by up to 500 times over the next decade, and an estimated $80 \%$ of it is still going into landfills and incinerators.

Digital devices are scrapped at an alarming rate instead of being salvaged, fixed, and reused, thus losing their final product added value. At economic European level, there is strong market demand across Europe for quality second-hand electronic goods. On average, 50\% of people in Europe according to a survey by Flash Eurobarometer [4] — would be happy to buy a second-hand appliance. But in Europe less than $40 \%$ of waste is properly treated. Reuse is a significant employment opportunity: it has potential to employ 10 times 
more people per ton of material processed than recycling activities. The WEEE Directive (2012/19 EU)[6] is trying to solve this problem by fixing a minimum quota of $5 \%$ for reuse treatment. But issues such as insufficient software tools to trace the EEE, problems with uniquely identifying digital devices and their components, and difficulties in accounting for reuse make it impossible to know if a device is finally counted twice as reused and recycled. We also cannot guarantee if a reused device is recycled, and where.

Public authorities are willing to promote the positive aspects of reuse, but informal recycling of electronics in the developed and developing world has emerged as a new global environmental concern. Without a traceability solution and a commitment from consumers of reused products, it is uncertain if donated digital devices for reuse may end up being exported illegally and potentially polluting the environment. Such risk and slippage is the main drawback in the promotion and the practice of reuse in public and private organisations or individuals.

Our proposal is to enable direct donations of devices that are up-to-date and to guarantee that any reused device is finally properly recycled. This is supported by a set of open-source support tools and a distributed Internet platform that result in an ecosystem for direct donation of devices. Devices are prepared for reuse with traceability in donor's locations, as depicted in Figure 1. This minimizes losses because there is no need to transport or centralize the EEE. Social enterprises (preparators) active in reuse and repair are not excluded from this ecosystem. In our pilots, donors mostly delegate preparation for reuse tasks (hardware rating and testing, deletion of data, inventory, labeling, and packaging) to reuse centers. Preparators take only products that need to be repaired, or refurbished and recycled if they are authorized collectors.

\section{Reutilitza.CAT - Platform Case Study}

A team of volunteers, activists, and researchers at the Universitat Politècnica de Catalunya has created eReuse.org, an eReuse platform for digital devices. eReuse is an umbrella website that federates several specific eReuse programs and provides a set of open-source software tools 11 To illustrate this concept, we present Reutilitza.cat as an example of a local eReuse ecosystem in Catalonia (Spain), where public organisations, private companies, and recipients exchange services and goods. This platform is in close cooperation with the public waste agency, associations of manufacturers responsible for recycling 80\% EEE in Spain, social enterprises that repair and refurbish equipment, and consumer groups guaranteeing the final recycling of the devices. eReuse.org has six specific programs in incubation. The oldest and most developed is Reutilitza.cat. With 659 registered users, it has facilitated the donation of 1640 digital devices to 891 social initiatives and registered so far more than 2,200 devices for eventual reuse (June 2015).

\footnotetext{
${ }^{1}$ https://github.com/eReuse
}

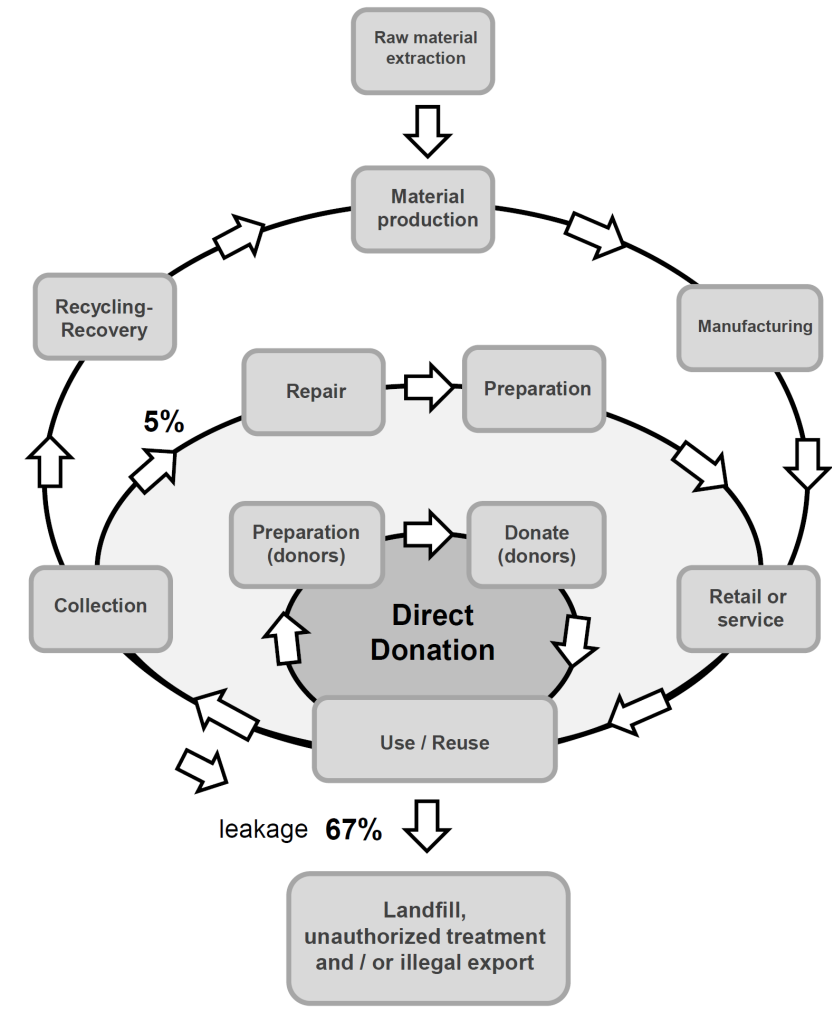

Fig. 1. Direct EEE donation embedded in circular flow of materials economy

One of the most active activities in Reutilitza.cat is the CoreTIC program, a pilot project performed for the Catalan Government of Catalonia to test the procedure for reuse of office computers, with a potential of more than 30,000 reusable computers per year ${ }^{2}$. This initiative has contributed 1,496 devices registered in the Reutilitza.cat platform, of which 1,212 (see Table I) had adequate specifications, passed hardware tests, and were collected from receivers (NGOs, social institutions) in the donors' location. This implies that about $80 \%$ of the devices that become obsolete in the government are still reusable, but this is just one sample from a public organisation.

This case study presents the tools of our current prototype, and the results of the pilots on how they contribute to donors, receivers, and reuse centers to gain effectiveness, efficiency, and traceability to ensure recycling. Specific objectives have been overcome such as accessing sufficient volumes of goodquality used devices, preparing first those which are traceable and with more potential for reuse, and involving receivers in the collection. Future case studies should evaluate the improvements that tools have achieved over operation times, social impact (digital literacy) and environmental impact (device lifetime extended) of these donations, the value generated by donors (such as employee satisfaction, engagement on social media), and the proportion of donated equipment that is finally recycled.

${ }^{2}$ Data sets for Reutilitza.cat pilot on CoreTIC Catalan Government program: http://data.ereuse.org/papers/devices-coretic.xlsx 


\section{A. Registration for traceability of digital devices}

Traceability is key to ensure that reuse contributes to recycling and to avoid that an extended lifetime of a device results in uncontrolled waste. The design of the process and the support tools should be proposed and discussed with stakeholders, and data generated should be open and standardized to help in the evaluation of how to minimize the environmental impact by extending the useful life span and ensuring final recycling of digital devices.

There is a lack of global traceability services for EEE that assure donors that their equipment will not end up polluting. Lack of traceability is the major impediment for the reuse sector to flourish. Traceability is required to enable public and private donors, public governments, receivers, and reuse centers to be the main consumers and promoters of this service. Donors fear that after reuse, their electronic devices may not end up being recycled. This could severely impact their image if such devices pollute the environment. Traceability should not be perceived as an obligation but as a way to optimize the reuse process and prevent e-waste leakage.

To add traceability to EEE, we must register a unique global identifier for all the devices and its components. In Reutilitza.cat we use the serial numbers of the components (and the MAC network card when available), which we collect using a custom software installed in an Ubuntu Linux live USB/CD. During the registration process, a Unique Device Identifier (UDI) is generated using the software lshw (Hardware Lister) providing detailed information on the hardware configuration of the device. The time needed to extract this information has a median of 44 seconds and a mean of 31 seconds per device (see Fig. 3). It can report exact memory configuration, firmware version, main board configuration, CPU version and speed, cache configuration, bus speed, etc. The data is used to rate the hardware to decide if it should be prepared for reuse. It also contains the serial numbers of components that we suggest to be used as global identifiers, as shown in Table 1 We achieved in our pilot a high success rate in automatically capturing these hardware characteristics and serial numbers; e.g. $99.3 \%$ for network card and hard disks, and $87 \%$ for manufacturer.

TABLE I

Testing Unique Device IDENTIFIERS

\begin{tabular}{lrrrrrrr}
\hline Serial\# & \multicolumn{6}{|c}{ Serial numbers of internal components } \\
\hline & UDI & Net & \multicolumn{2}{c}{ HD Manufact. MotherB. } & RAM & CPU \\
\hline Detected & 1212 & 1203 & 1204 & 1055 & 783 & 1187 & 1049 \\
\hline Non Detected & 0 & 9 & 8 & 157 & 429 & 25 & 163 \\
\hline \% Detected & $100 \%$ & $99.3 \%$ & $99.3 \%$ & $87 \%$ & $64.6 \%$ & $97.9 \%$ & $86.6 \%$ \\
\hline Unique & 1212 & 1203 & 1171 & 1028 & 729 & 179 & 22 \\
\hline Not unique & 0 & 6 & 33 & 27 & 54 & 1008 & 1016 \\
\hline Unique (\%) & $100 \%$ & $100 \%$ & $97 \%$ & $97 \%$ & $93 \%$ & $15 \%$ & $3 \%$ \\
\hline
\end{tabular}

UDI is a Unique Device Identifier. The algorithm used for generation is as follows: UDI = (Manufacturer Name + Manufacturer $\mathrm{S} / \mathrm{N}$ ). As seen in Table 1 the manufacturer serial

\footnotetext{
${ }^{3}$ Hardware Lister (lshw): http://www.ezix.org/project/wiki/HardwareLiSter
}

\section{Serials Unique}

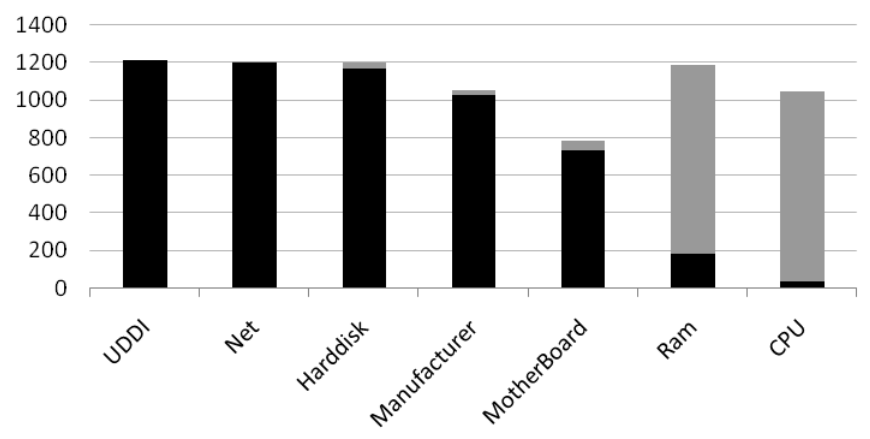

Fig. 2. Ratio of unique serial numbers in computer components

number for diverse components is automatically detected in $87 \%$ of cases, and from these $97 \%$ is unique (Figure 2). If not obtained automatically, it can be provided manually by the human operator registering the device defined as (manufacturer + id manufacturer) or derived from a combination of serial numbers from key components. This is in addition to traceability at the component level.

The data is stored locally or sent automatically to the Reutilitza.cat website, and from there it is reported to eReuse.org. Data is signed with an eReuse signature to guarantee that it has been created by the eReuse software and that a malicious user has not tampered with it. After the device is registered, the donor tags the computer by printing its identifier and QR code that points to the uniform resource locator (URL) where the device description is stored. A smartphone can be used to geotag and visualize the characteristics of any device. A QR code is more consumer-friendly than a RFID tag because mobile phones with built-in cameras can read the tags. This allows for a direct connection between reuse consumers and traceability information. A prototype mobile application performs the main functions on a computer: geolocation, and changing states (donated and recycled).

\section{B. Reusability potential and preparation for reuse}

Tools can assist in the certification of the reusability potential of digital devices. This improves efficiency, as only products with a high potential for reuse may be donated, and the remaining will be recycled. These tools can ensure hardware rating, deletion of data, tests, and inventory at the component level for a fleet of digital devices, even remotely. In our experience, productivity must be at least 10 devices per operator and hour to be cost effective and therefore sustainable.

Hardware rating is done instantly after the registration process. The duration of the erasure of data depends on the intensity level the donor selects. A high intensity data deletion is around $0.2 \mathrm{~GB}$ per second. Finally, computers are left operational by installing a free operating system that fits the characteristics of the device. By default, our tool uses a lightweight version of Ubuntu (Lubuntu). Our next challenge 


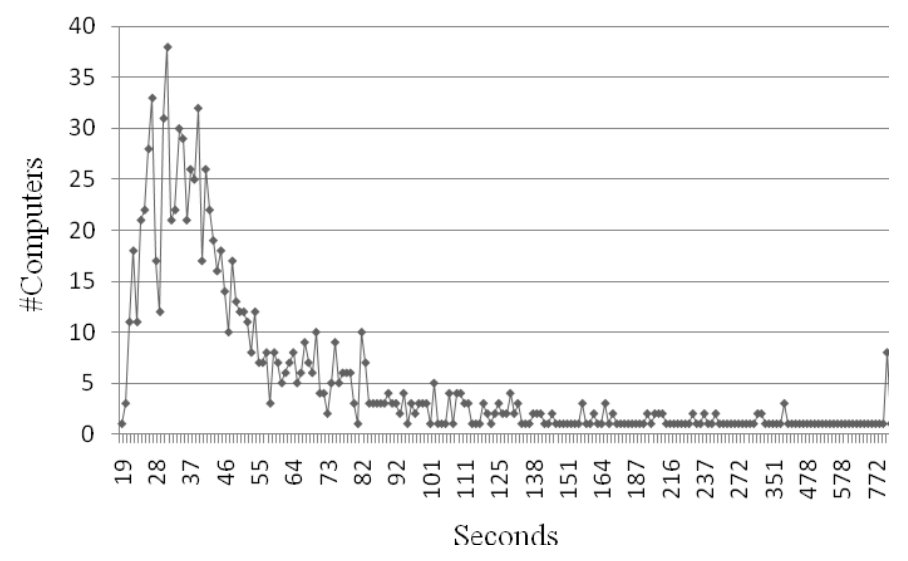

Fig. 3. Time for registering devices

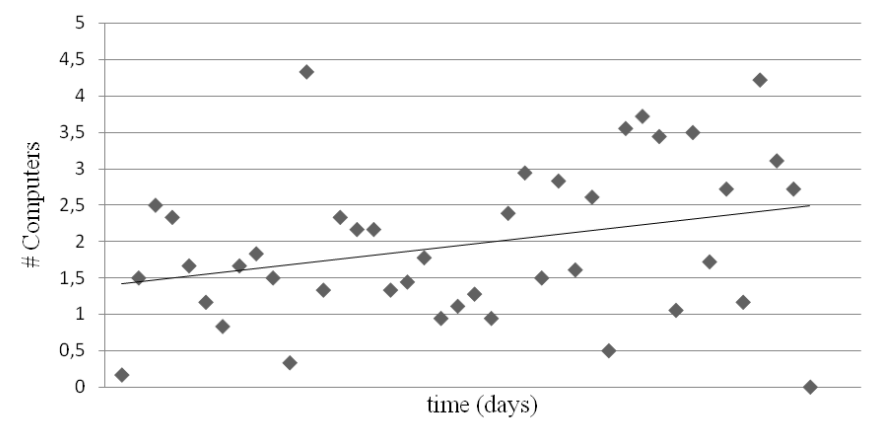

Fig. 4. Number of computers prepared by operator per hour

is doing all these steps in an unattended and parallel way. For that, we are currently testing a network boot-based tool.

Figure 4 shows that during a one-week period an operator managed to prepare for reuse at a rate of 2.5 devices per hour. This also includes testing the keyboard, screen, and mouse.

The steps performed in our pilot to prepare a device for reuse were the following:

- T1: Selecting the device to register and transfer to the registration area. The devices were on pallets and were moved between 10 to 70 meters.

- T2: Unpacking towers, monitors, keyboards, and mice. The devices were packaged in cardboard boxes.

- T3: Booting the live-CD system (Ubuntu) in text mode. The devices were located on different tables that allowed processing up to four devices at a time. Power cables and monitors were not changed to save preparation time. Text mode was less time-consuming than graphic mode.

- T4: Running hardware detection to read all computer components (characteristics, serial numbers). The registration program starts directly and automatically after boot.

- T5: Entering particular parameters of the devices to allow integration with donors' inventory.

- T6: The file was generated, signed, and uploaded to the
Reutilitza.cat site.

- T7: Generating and pasting QR labels. The labels had to be printed in another space (5-10 minutes away). The printing process was done every 30-40 devices registered.

- T8 Testing monitor, keyboard, and mouse. Monitors were tested with a Microsoft Windows environment.

- T9: Packaging and storing devices on the pallets. This process was the most time-consuming. The operators had no previous experience in packaging.

- T10: Other activities (unexpected issues)

\section{Current Work: Platform and architecture}

The architecture of the reuse platform is being refined and extended so it can be instantiated by multiple organisations and integrated in the eReuse.org website, and to provide an overall picture of aggregated data sets to facilitate the analysis and traceability audit. Platforms are federated and autonomous, and report data to eReuse.org. eReuse should collect from each device, at least, the geographical paths it has followed (not in detail to preserve privacy), the reuse platforms where it has been stored, the collection point where it was located before its final recycling and, in the case of computers, the history of its components.

Platforms provide to donors a set of support tools to do themselves the task of preparation and certification of devices for reuse (deletion of data, tests of operation, inventory, labeling, finding recipients, and packaging), and to receivers, the support tools to request devices, track them, and finally recycle them on authorized points.

Social enterprises, and reuse and recycling centers can create their own platform instances or operate one as a cloud service. Reuse centers should find clear pathways for replicate up the models, offering to donors services such as preparation for reuse tasks and to social recipients maintenance services equivalent to an extended guarantee.

Technology Readiness Levels (TRLs) indicate the maturity level of particular technologies. They provide a common understanding of technology status and address the entire innovation chain. TRL has been defined by the Horizon 2020 Research and Innovation programme [8]. Our current solution is at TRL6, and during 2015 and at the beginning of 2016 we plan to reach TRL7 with a grant from the "Collective enHanced Environment for Social Tasks" (CHEST) project as part of the "Collective Awareness Platforms for Sustainability and Social Innovation" (CAPS) initiative.

The main functionality being developed (new, reimplemented, or extended from the pilot) is:

1) Preparation for reuse toolset: A set of tools to support the process of preparing for reuse and/or tracking of digital devices that essentially consists of: erasing data, tests and operation performance, inventory, labeling, and packaging. It is fundamental to develop a mobile application to help in the tracking, using QR codes generated during the certification process as seen in Figure 6

2) Device Inventory Management - External Transfer: At the time of the transfer or the donation of devices, the donor 


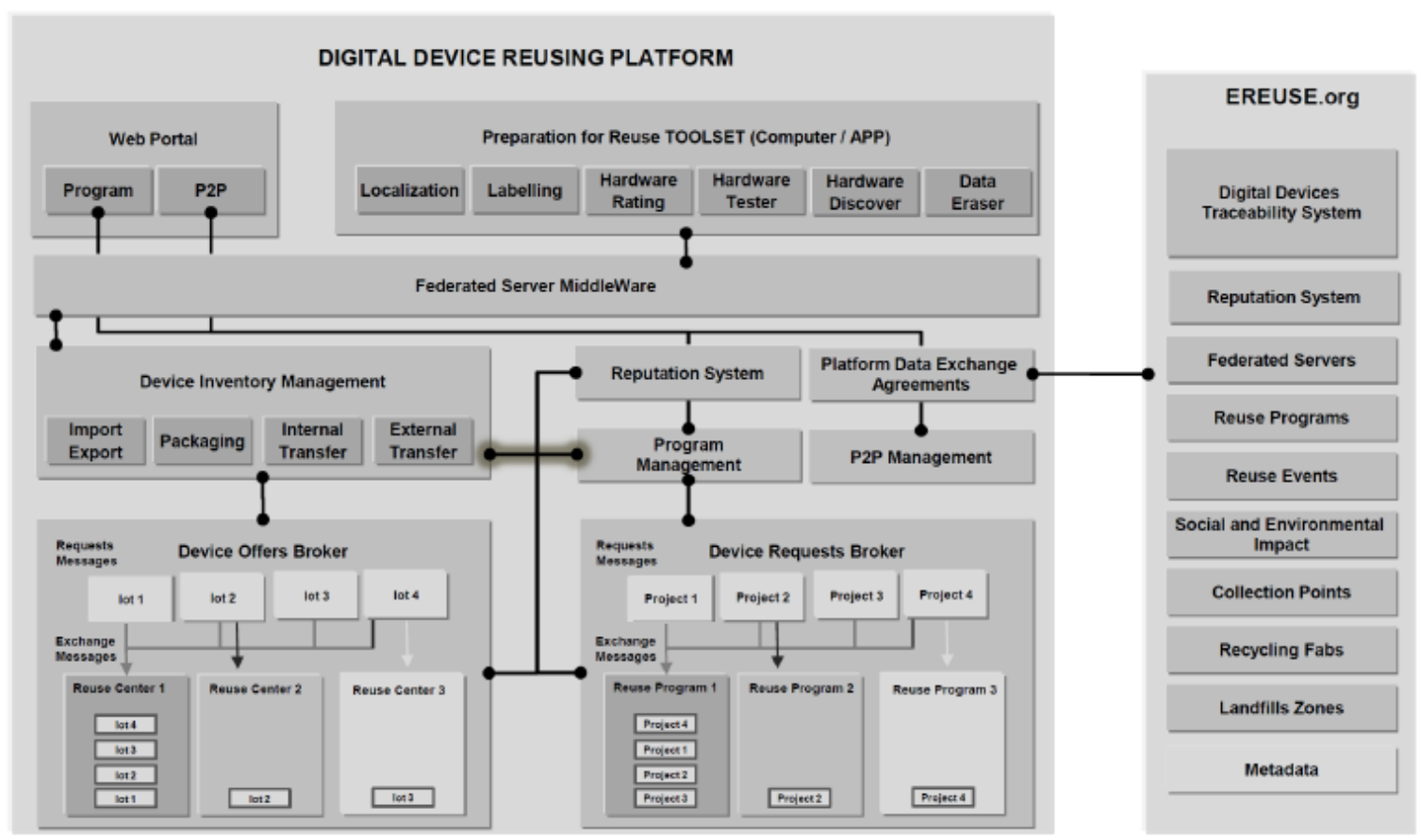

Fig. 5. Platform and eReuse.org architecture

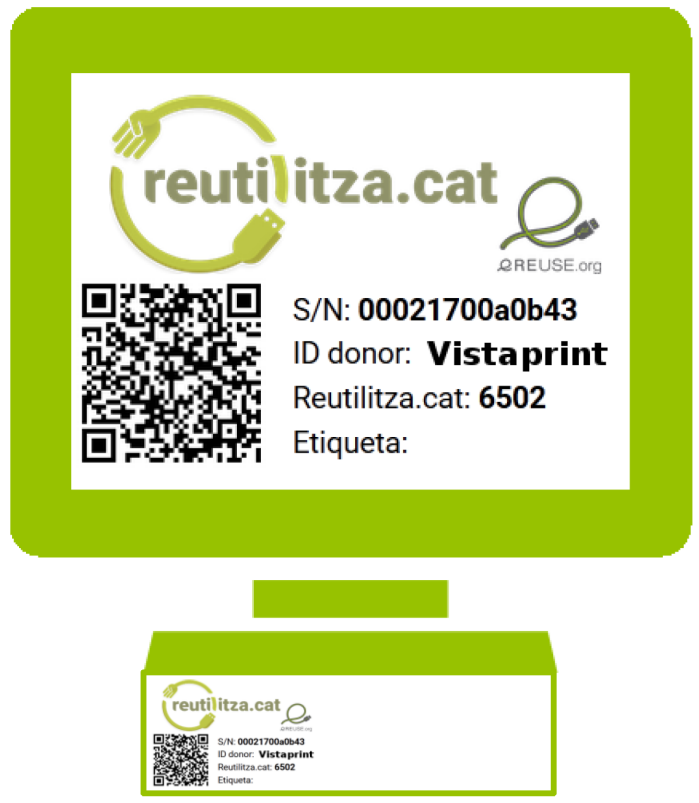

Fig. 6. Screenshot of a computer label

must enter a number in a donation application that shows where devices are located (in the warehouse) and a list of devices with their identification number. After that, the donor reads the QR tag of all the devices to be donated. The transfer is done once all devices have been read. Once the transfer is accomplished, the receiver gets an email indicating the steps to take to perform the commissioning and sign a legal document with the ownership and responsibilities assumed by

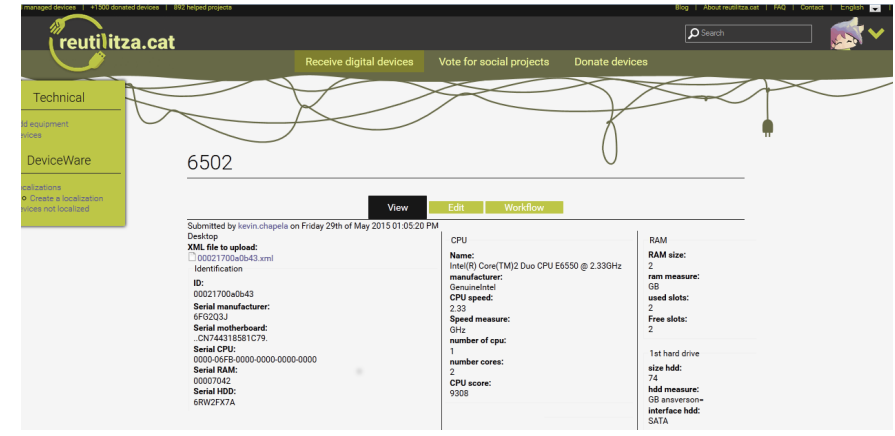

Fig. 7. Inventory information for a device

the receiver (including its final recycling). Figure 7 shows an example of the inventory information collected by the Reutilitza.cat web portal.

3) Device Offer Broker: A broker among donors and reuse centers. Publishers (donors) make requests for preparation for reuse. Can be many unregistered devices (eg. 20 devices model $\mathrm{X}$ fully operational). Reuse centers are subscribed to receive these requests. Once conditions are accepted and details are arranged, reuse support staff members go to donors' locations to prepare the devices for reuse. Reusable devices are stored at donors' locations, and non-reusable devices may be collected by staff from reuse centers for direct recycling.

4) Device Request Broker: A broker among donors and receivers. Donors select projects that have reached a threshold of reputation (e.g. by recycling previously donated devices, projects with social impact, social support from beneficiaries). 


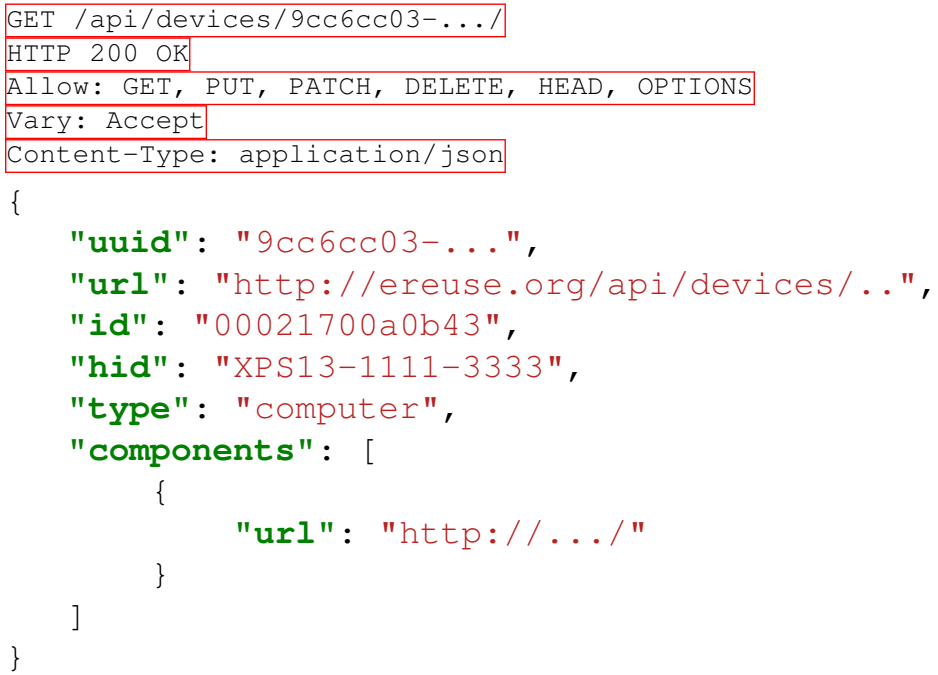

Fig. 8. REST API to access device details

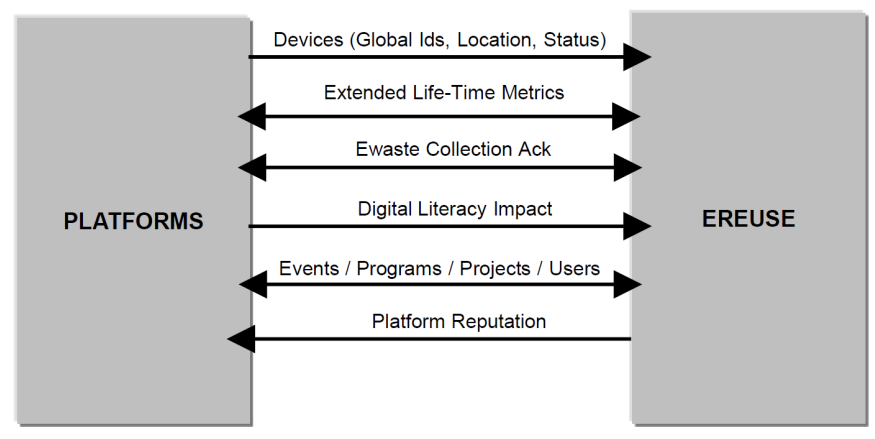

Fig. 9. Data Exchange between platforms and eReuse

Receivers should: (1) fill out the required fields and select social impact indicators, (2) accept or reject (online) the devices assigned, (3) collect them on the donors' location, (4) publish a post on social networks to announce the donation, (5) geo-locate the device for tracking, (6) use the devices, extending their life-span, and (8) once the device is no longer needed, and if it still works, they should offer it again for reuse to the platform, and if not, they have to send it to an authorized collection point for recycling and geo-locate it there for tracking that final step in the life of the device.

\section{A. Data exchange with eReuse.org systems}

A data exchange protocol must be standardized to facilitate traceability beyond the known agents in the reuse chain. Our suggested approach combines the protection of personal or private information with Open Data information aggregated in a common federated name space for all eReuse local platforms. This is being defined as a REST+JSON API as follows:

Our data should be standard, and at least part of it should be open while preserving privacy, so meanwhile the systems inside the ecosystem of eReuse can operate, and external projects can use the overall Open Data in new and innovative

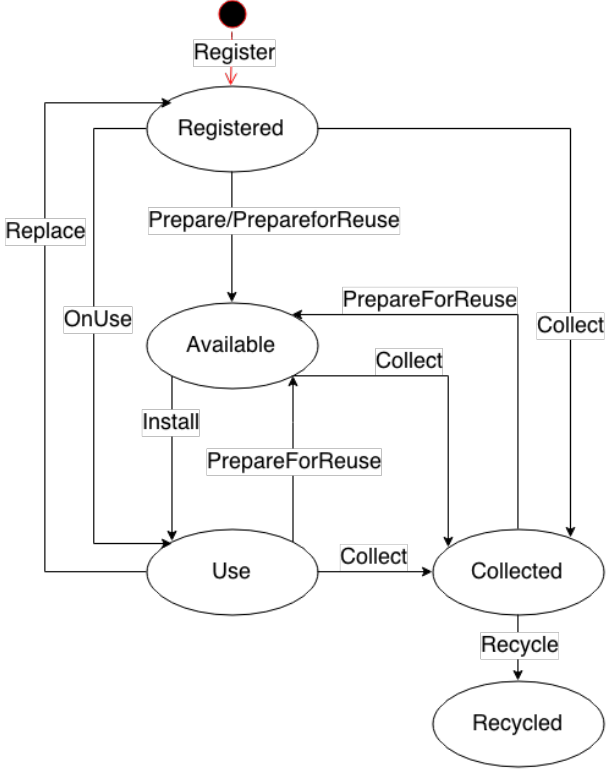

Fig. 10. Device Life Cycle

ways such as research or audit. In the lack of a standardized ontology regarding our ecosystem, we plan to create and expand one, constructing it using parts of existing and well-known ontologies. Regarding the technical part of the exchange of information, following our view to use simple and standardized architectures and languages, we are going to use REST interfaces with JSON data structures, in concrete JSON-LD when we want to represent knowledge.

\section{B. Digital device life cycle}

A platform record major states in the lifecycles of devices and reports them to the eReuse.org repository. The main states are: registered (not in use), available to be used (and reused) in a workplace, replaced (e.g. for failure, obsolescence) but not yet discarded, catalogued to be reused or recycled.

To increase the guarantee of final recycling, two actions are suggested: i) first, platforms should geo-tag all authorized collection points and ask receivers to perform a geo-location action once they send devices to recycle. If last geo-location is performed within the geographical area of known collection points, this provides a high confidence degree that devices are collected, and ii) second, reward recipients with reputation points to increase their chances to renew devices if they succeed previously in extending the lifetime of devices and collaborate in its traceability.

\section{Open Knowledge Base}

\section{A. Life-Time Extension}

The information in the eReuse lifecycle repository should allow deduction of the:

1) Number of times: a device has been reused.

2) Durability or elapsed time: between first and last usage. 
3) Running time: The total time the device has been operating. This information is provided automatically by users to platforms using programs installed on devices. To preserve the privacy of users, eReuse only collects the total time. This information is useful for the platform to rate the reputation of receivers.

4) Usage time: The total time the device is in usage status. The time between each cycle of use (reuse) should be minimized (e.g. when devices are registered waiting to be prepared for reuse but not in use, or waiting to be commissioned in the user's workplace). This information is useful for benchmarking across different platform instances.

\section{B. Electronic Waste Landfills}

A person that finds electronic waste that has been monitored by eReuse can notify eReuse about it and its location. What information must be notified and in which part of the product can it be found? Devices that have been managed by platforms implementing eReuse data exchange protocol should have a QR tag that uniquely identifies each device. If this label does not exist, internal component labels can be used to find out the identity of the computer (all components should have one) or if a component stills works, this information can be extracted by software programs that read hardware meta-data. The person using an application can notify this identification data and the location of the landfill. eReuse does not have information about the identify of its last owner but knows which platform instance was managing this device. To resolve the incident, eReuse notifies the involved platform about it and follows-up on the measures taken by the platform to address it.

\section{Corporate Social Responsibility (CSR)}

The main challenges to overcome for advancing reuse are that involved agents communicate its social and environmental value, and that risk from pollution and economic costs is minimized. Agents involved in reuse must make it attractive, and donors should be rewarded enough (public image and traceability) and operating costs should be optimized. The platform should enable donors to capitalize a value in CSR in excess of that amount, following a crowdfunding model. Companies can choose from a range of projects to donate their devices, and this allows them to target that local audience with ads and promotional campaigns.

\section{Data for Circular Economy}

Our main contribution is the creation of value, and access to an aggregate of open data. with details about traceability and social impact of reuse. Many programs measure success from the number of donated devices, but few would allow traceability and impact monitoring. The value generated by reuse is not sufficiently communicated, and it is not possible to assess the value due to lack of data. Existing initiatives could be more rigorous in terms of tracking if they included in their platforms aspects of traceability such as the registration of certified hardware and notification of their geographical traceability.

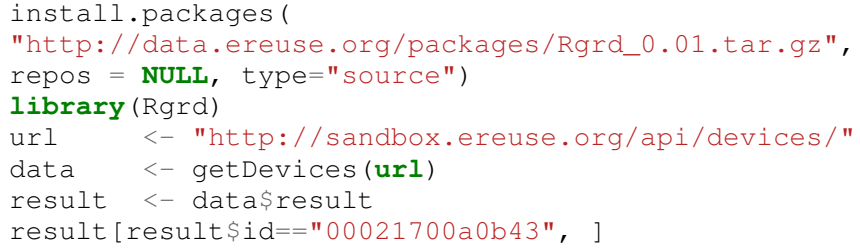

Fig. 11. R code fragment to interact with the REST interface

To facilitate the understanding of the value generated, we describe the process of data analysis. Ereuse platforms exchange traceability data about the devices they manage (see Figure 7). This information has been transferred from Reutilitza.cat (the platform) to ereuse. eReuse receives traceability data from each platform and implements the protocol. It provides knowledge of device recycling locations and reveals the durability and total usage time. This builds confidence in donors, governments, manufacturers, and donor organisations, and is in line with recent European directives. Most important, it generates data that enables (building the knowledge base for environmental action and sustainability) movement toward a fully circular economy. For that purpose, eReuse allows accessibility to this information via a REST interface (Figure 8). Access to eReuse data can be accomplished from the platform, but also from a web browser or programmatically, as shown by the $\mathrm{R}$ programming language fragment in Figure 11. This is part of the Rgrd eReuse software package.

\section{RELATED WORK}

Computer reuse through donation is being conducted by other initiatives and organisations. Several social enterprises are active in this area. One of the most well known is ComputerAid.org, a not-for-profit organisation in the UK and Kenia founded in 1998 to bridge the digital divide by providing refurbished PCs from the UK to educational and nonprofit organisations in developing countries. Computer Aid has provided over 200,000 refurbished computers to educational institutions and not-for-profit organisations in more than 100 different countries to date. Computer Aid offers a decommissioning service to UK companies, government departments, and universities that are upgrading their computer systems. Donated PCs are shipped to their central workshop in the north of London where they are data-wiped, refurbished, and tested. Non-profit organisations in the developing world can apply for refurbished computers and are charged a handling fee of $£ 42$ plus shipping.

Labdoo.org is an "humanitarian social network to help bring education around the globe". The goal of the social network is to send unused laptops, ebook readers, tablet-PCs, and any device that can be loaded with education software to needy schools around the world using collaboration and without incurring any additional economic or environmental costs.

To date, Labdoo has delivered more than 2,700 devices to more than 450 schools. Similar to reutilitza.cat, it has a web platform that allows direct donation. In the case of Labdoo, part of these donations are international, so the organisation 
developed the logistics to take advantage of people travelling to destinations so they can carry the devices without generating an extra environmental burden. Labdoo has an active social network, a collaborative wiki, and a very committed community to coordinate the transfer of devices, with more than 120 hubs where donors can learn how to properly prepare the equipment for donation.

Both initiatives, among many other organisations and programs, can benefit from the tools, process, open data repositories, and external audit of the process that eReuse provides. Our tools allow an automated and certified preparation of devices for reuse, and automatically report to eReuse.org traceability data about devices. We are in talks with Computer Aid and Labdoo so they can participate in eReuse.org. Specifically, the tools that perform automated and signed reading of hardware information (serial numbers) and information exchange protocol for the platform can report eReuse traceability information.

\section{CONCLUSION}

We propose a model and a set of open-source, mature, decentralized, local, scalable technologies to enable direct donations of those digital devices that are up-to-date and have local demand willing to collect it at the donor's location and ensure final recycling.

Preparation for reuse and donation in donor's locations reduces costs and minimizes losses because there is no need for centralized logistics for the EEE. This increases efficiency because the preparation can be done in an unattended way and without the need for additional steps of opening or powering up the computer. This also increase engagement of donors because they know directly who are receiving these devices.

An evaluation of a process design and tools for preparation for reuse and to ensure traceability has been presented and evaluated with an extensive pilot during more than four years and with two thousand devices reused. The improvement of technology to assist in the certification improves efficiency, as only products with a high potential for reuse may be donated, and the remaining will be recycled. The time required to run a software tool to register and select devices with the required characteristics is minimal. We had a high success rate in automatically capturing these hardware characteristics and serial numbers. This suggest that our approach to create Unique Device Identifiers based on internal components and also perform traceability by component can be considered a successful model.

We are presenting for discussion our current development of a distributed ecosystem of federated and autonomous platform instances. These instances manage and report data to eReuse servers that are responsible for storing the geographical path of devices along reuse and until recycling. To discourage or avoid collusion and increase the guarantee of final recycling, we suggest two actions: first, geo-tagging all authorized collection points and asking receivers to record locations once they deliver devices for recycling; and second, awarding recipients with reputation points if they extend the lifetime of devices by using reused devices and if they contribute to traceability, as these reputation points are exchangeable for future reused devices.

Data reported by platforms can create an open knowledge base with indicators per region where platforms can benchmark their reuse performance and efficiency in selecting the most appropriate donors, reuse centers, and receivers. This will facilitate the process and increase the life and usage time of devices. Citizens can analyze data about hardware tests and device and component durability, and report about electronic waste landfills.

Ongoing case studies should evaluate the improvements that support tools bring in processing/operation times, social impact (digital literacy) and environmental impact (extended device lifetime) of donations, the value generated by donors (e.g. employee satisfaction, engagement on social media), and the proportion of donated equipment that is finally recycled.

\section{ACKNOWLEDGMENT}

This work is supported by the European Community Framework Programme 7, Collective Awareness Platforms for Sustainability and Social Innovation (CAPS), project "Collective enHanced Environment for Social Tasks" (CHEST), contract 611333.

\section{REFERENCES}

[1] Baldé, C.P., Wang, F., Kuehr, R., Huisman, J. (2015), The global ewaste monitor - 2014, United Nations University, IAS - SCYCLE, Bonn, Germany.

[2] D. Franquesa, J.L. Cruz, C. Álvarez, F. Sánchez, A. Fernández and D. López, The social and environmental impact of engineering solutions: from the lab to the real world, Int J Eng Educ, vol. 26, Issue 5, 2010, pp. 1144-1155.

[3] A. Cerrillo. Residus electrònics fora de control. La Vanguardia. 29.05. 2014. pp. 28-29.

[4] Eurobarometer, 2014. Flash Eurobarometer 388, Attitudes of Europeans towards Waste Management and Resource Efficiency, European Commission. Available at: http://ec.europa.eu/public_opinion/flash/fl_388_en.pdf

[5] Directive European Commission MEMO/08/764, Questions and answers on the revised directive on waste electrical and electronic equipment (WEEE), December 2008, pp. 1-6.

[6] Directive 2012/19/EU of the European Parliament and of the Council of 4 July 2012 on waste electrical and electronic equipment (WEEE). July 2012.

[7] M. Schuelp, C. Hagelueken, R. Kuehr, et al., Recycling from e-waste to resources, United Nations Environ Program United Nations Univ, 2009.

[8] Horizon 2020 programme, Technology readiness levels (TRL), European Commission, July 2014. 